\title{
Las finanzas éticas como instrumento al servicio de la economía solidaria. La experiencia de Coop57
}

\author{
Ethical finances as a tool of solidarity economy. \\ The experiencie of Coop 57 \\ Raimon GASSIOT BALlBÈ \\ Coordinador adjunto Coop 57 \\ raimon@coop57.coop
}

Recibido: $15 / 11 / 2012$

Revisado: 27/11/2012

Aceptado: 16/02/2013

Disponible on line: 16/04/2013

\begin{abstract}
Resumen
Este artículo analiza el papel de las finanzas éticas como un instrumento al servicio del desarrollo de la economía solidaria, una economía que trata de satisfacer las necesidades de las personas, tiene una gestión democrática, distribuye equitativamente la riqueza generada e incorpora como principios básicos criterios de sostenibilidad social y medioambiental. Hace un breve análisis de la actual crisis y del papel que en ella ha jugado el sistema financiero convencional, propone la economía solidaria y las finanzas éticas como alternativa con potencial de transformación social y se centra en explicar la experiencia de la cooperativa de servicios financieros y solidarios Coop57. Para acabar, propone algunas ideas sobre cómo desde la disciplina y la práctica del trabajo social se puede contribuir al desarrollo de la economía solidaria.
\end{abstract}

Palabras clave: economía solidaria, finanzas éticas, transformación social, cooperativismo, autogestión, mutualismo.

\begin{abstract}
This article analyzes the role of ethical finances as a tool for development of the solidarity economy, an economy that seeks to satisfy the needs of people, has a democratic governance and an equitable distribute of wealth generated, and incorporates principles of social and environmental sustainability.

It makes a brief analysis of the current crisis and the role it has played the conventional financial system, proposed the solidarity economy and the ethical finances as an alternative with potential for social transformation and focuses on explaining the experience of the cooperative financial services Coop57. Finally, proposes some ideas of how the discipline and practice of social work can contribute to the development of solidarity economy.

Keywords: economic solidarity, ethical finances, social transformation, cooperative movement, self-management, mutualism.
\end{abstract}

Referencia normalizada: Gassiot Ballbè, R. (2013): «Las finanzas éticas como instrumento al servicio de la economía solidaria. La experiencia de Coop57». Cuadernos de Traajo Social, 26(1): 75-85.

Sumario: 1. El papel de las finanzas convencionales en la actual crisis económica. 2. Las finanzas éticas como alternativa. 3. La experiencia de Coop57. 4. Líneas de futuro. 5. El Trabajo Social y las finanzas éticas y solidarias. 6. Referencias bibliográficas.

\section{El papel de las finanzas convencionales en la actual crisis económica}

La actual crisis económica (que, de hecho, tiene todas las características de una crisis sistémica de largo alcance), pone de relieve los pro- fundos desajustes del sistema económico y social capitalista y, sobre todo, la necesidad de buscar alternativas basadas en criterios como la solidaridad, la equidad, la justicia, la democracia, la autogestión y la ayuda mutua. 
La crisis tiene un origen eminentemente financiero. A pesar de que este artículo no pretende hacer un análisis pormenorizado de cómo se ha convertido el sistema financiero en el eje de nuestra economía, una economía fundamentalmente especulativa y sin base real en la actividad productiva (producción e intercambio de bienes y prestación de servicios), sí que creo interesante apuntar algunas ideas clave que nos ayuden a interpretar la realidad para poder plantear alternativas. La primera es que hace ya mucho tiempo que las finanzas han pasado de ser un medio para facilitar el ahorro y para financiar las actividades económicas y determinadas necesidades de consumo de las personas (como la adquisición de viviendas, en la medida que implican un desembolso importante de dinero) a ser un fin en sí mismas. En ese tránsito, el objetivo ha pasado a ser la maximización de beneficios a cualquier precio (no poner en contacto el ahorro y el préstamo), hecho que ha comportado un gran crecimiento del dinero y de la ingeniería bancaria, con derivados financieros de gran complejidad pero con una base muy precaria. Así, la pérdida de base real de buena parte de la actividad financiera y el crecimiento de una inmensa burbuja especulativa estaban servidas. Y todo ello combinado, en nuestro país, con unos elevadísimos niveles de endeudamiento, tanto de las grandes empresas, como de las familias (enmascarando así, con el recurso al endeudamiento, una pérdida real de poder adquisitivo de la mayoría de la población respecto a una oferta creciente de bienes y servicios $y$, sobre todo, de un inmenso parque de viviendas a precios astronómicos) $\mathrm{y}$, paradójicamente, de las propias entidades financieras.

En este contexto, ha sido especialmente llamativa la bancarización de las cajas de ahorros. En poco tiempo ha culminado un lento proceso de hondas raíces, que ha comportado la extinción de unas entidades con arraigo local que originariamente tenían la función de promover el ahorro popular y de financiar la economía real de su ámbito territorial de actuación. Su desaparición formal es la certificación de su bancarización previa: hace ya tiempo que en la práctica su actividad se diferenciaba muy poco de los bancos privados, más allá de la persistencia de las obras sociales y de unas perversas ingerencias y fidelidades políticas.
La desaparición de las cajas deja un vacío que podrían cubrir las finanzas éticas, recuperando sus finalidades originarias (arraigo local, promoción del ahorro popular y financiación de la economía real), pero incorporando elementos específicos para evitar una evolución similar a la que han sufrido las cajas:

- Uso de criterios éticos y sociales para definir su actividad y su política crediticia y de inversión.

- Gestión realmente democrática y participativa.

- Control social efectivo.

Este último aspecto, estrechamente vinculado con el precedente, no debe confundirse con el control político; precisamente el control político-partidista ha sido una de las causas de los desmanes de las cajas de ahorros: inversiones faraónicas en obras públicas para mayor lucimiento del político de turno, sin ninguna lógica económica ni social (por ejemplo, aeropuertos desde los que no ha salido ni llegado ningún vuelo comercial), reparto de cargos en los consejos de administración para contentar distintas familias o determinados favores políticos, etc. Se trata de ver cómo la sociedad civil organizada puede mantener un control social efectivo sobre unas instituciones financieras que juegan un papel clave en nuestra vida económica y social.

\section{Las finanzas éticas como alternativa}

En el movimiento de las finanzas éticas existen distintas corrientes y tendencias. Desde entidades como Coop57, nos identificamos con aquéllas que conciben las finanzas éticas no solo como entidades con criterios éticos de inversión, pero que no replantean aspectos como el modelo de propiedad o de gestión de las propias entidades financieras, sino como un instrumento que contribuya a erigir un modelo económico y social alternativo al hegemónico. La actividad financiera debe ser sólo un medio para transformar la sociedad en base a los planteamientos de la economía solidaria, una economía que, para sintetizar, debe reunir las siguientes características:

- Estar al servicio de la satisfacción de las necesidades de las personas, en vez de estar las personas al servicio de supuestas necesidades económicas. 
- Distribuir equitativamente la riqueza generada. cipativa.

- Tener una gestión democrática y parti-

- Incorporar en toda su actividad criterios de sostenibilidad social y medioambiental, que aseguren que no se hipoteque el bienestar de las futuras generaciones.

La finalidad de las finanzas éticas debe ser promover el ahorro popular y, sobre todo, facilitar financiación a los proyectos y entidades de la economía solidaria para que puedan desarrollarse. No debemos perder nunca de vista lo importante, que la economía solidaria se desarrolle, crezca y tenga mayor incidencia económica y social. Sin ello, las finanzas éticas no tienen ningún sentido.

Otro aspecto fundamental es que los instrumentos financieros éticos deben incorporar los propios criterios de la economía solidaria: gestión democrática y participativa, propiedad colectiva, distribución equitativa de la riqueza generada, sostenibilidad social y medioambiental, etc. Sólo la aplicación práctica de estos criterios garantiza que las entidades de finanzas éticas se ajusten a su papel de instrumentos y no se alejen de su función originaria.

\section{La experiencia de Coop57}

El modelo de finanzas éticas que promovemos desde Coop57 pone el acento en el desarrollo de actividad financiera con criterios de arraigo local, proximidad y conocimiento de las entidades de economía solidaria. Esa proximidad con la base social es la que permite, por una parte, garantizar un control social efectivo de la entidad financiera $y$, por la otra, potenciar en lo local vínculos sociales y de vecindad que permitan construir de forma colectiva alternativas socio-económicas que nos ayuden a superar la crisis actual.

Coop57 es una cooperativa de servicios financieros éticos y solidarios que promueve el ahorro popular y destina sus recursos a financiar entidades y proyectos de economía solidaria con alto contenido social. No es un banco ni pretende serlo, desarrolla su actividad en base a principios cooperativos y de ayuda mutua. Con su sede social en Barcelona, lleva a cabo una actividad financiera como cooperativa de servicios y, como consecuencia, se rige por la
Ley de cooperativas. No tiene ficha bancaria y no está regulada por el banco central. Ello limita su actividad (no puede ofrecer préstamos a las personas o servicios de banca minorista, como por ejemplo cuentas corrientes, tarjetas de crédito, etc.), pero, por otra parte, le da mayor capacidad de autogestión y mayor agilidad para dar respuesta a las necesidades financieras de sus socios (las entidades de la economía social y solidaria) que las entidades financieras convencionales.

Coop57 no tiene clientes, tiene socios y socias, y sólo puede prestarles a ellos sus servicios financieros. En concreto, tiene dos tipos de socios:

- Socios de servicios: son entidades de economía solidaria (cooperativas, asociaciones, fundaciones, empresas de inserción, etc.). Pueden aportar ahorros y recibir préstamos de Coop57.

- Socias y socios colaboradores. Son mayoritariamente personas físicas que pueden ahorrar en Coop57, pero no recibir préstamos.

Para ser socias de servicios, las entidades que lo soliciten deben pasar por un proceso de evaluación ético-social para garantizar que cumplen los criterios de economía solidaria que tenemos establecidos. En este proceso de evaluación básicamente analizamos qué hace la entidad y cómo lo hace. Si uno de estos dos aspectos es claramente positivo y el otro no es negativo, la admitimos como socia. Pongo dos ejemplos sobre cada uno de estos aspectos. En qué hace, podríamos fijarnos en L'Olivera, una cooperativa de trabajo de integración social ubicada en Vallbona de les Monges, un pueblo de la provincia de Lleida afectado por un persistente proceso de despoblación, que produce vino y aceite de alta calidad, dando trabajo a personas con discapacidad intelectual y recuperando viñedos y técnicas autóctonas. Su actividad cumple claramente una función social y se gestiona en consonancia con los criterios de la economía solidaria.

Como ejemplo de cómo se lleva a cabo la actividad podemos poner a Mol-Matric, una cooperativa metalúrgica que desarrolla tareas de matricería para clientes que, en su mayoría, son multinacionales del sector del automóvil, el ferrocarril o la energía eólica. La función social de su actividad no destaca respecto a otras matrice- 
rías, pero sí cómo la desarrolla. Se trata de una cooperativa de trabajo creada en 1981 cuando, en el contexto de la crisis industrial de los años 1980, los trabajadores de una empresa mercantil en quiebra de Barcelona se apropiaron de sus instalaciones y la reflotaron de forma autogestionada. Gracias a una reinversión constante de los excedentes y a la implicación de sus socios, la cooperativa no solo ha mantenido los puestos de trabajo en las sucesivas crisis que han diezmado el sector industrial catalán, sino que ha creado nuevos, ha asegurado el relevo generacional y actualmente está abriendo una nueva planta. Y todo ello aplicando principios cooperativos y de democracia económica, participando en el movimiento de economía solidaria y destinando una parte de los excedentes a proyectos de solidaridad en Nicaragua, el Sahara o Etiopía.

Los dos tipos de socios (entidades socias y personas ahorradoras) son colectivamente los propietarios de Coop57 y, como consecuencia, pueden participar en las asambleas y en el resto de órganos sociales. De esta manera, además de poner en práctica la democracia económica, se supera la tradicional separación y contraposición entre los ahorradores y los receptores de financiación: todos juntos deciden las cuestiones importantes y las líneas de trabajo de la cooperativa.

Coop57 remonta sus orígenes a finales de los años ochenta y principios de los noventa. Inició su actividad en Cataluña a partir de la lucha de los trabajadores de la Editorial Bruguera para mantener sus puestos de trabajo. Cuando la editorial cerró definitivamente, un grupo de antiguos trabajadores creó un fondo con parte de las indemnizaciones que recibieron por su despido para promover proyectos económicos que persiguiesen la creación de puestos de trabajo de calidad, especialmente aplicando modelos cooperativos.

En 1996 con este fondo se creó Coop57. En un inicio, su desarrollo estaba vinculado con el cooperativismo de trabajo asociado, pero progresivamente fue ampliando su base social a otros tipos de entidades de la economía social y solidaria. Paralelamente, también fue creciendo su base de socios y socias colaboradores. A partir de 2005 Coop57 puso en práctica un crecimiento en red, a raíz del interés que había despertado este experimento en otros territorios. En aquel año, una red de entidades de economía social y solidaria de Aragón y Coop57 conjuntamente decidió compartir el proyecto. La idea fue aprovechar el modelo, la estructura jurídica y la estructura técnica de Coop57, pero aplicando un modelo que permitiese que cada territorio se autogestionase. Ello dio lugar a la creación de Coop57 Aragón en base a un planteamiento de desarrollo en red.

De acuerdo con estos principios, posteriormente se constituyeron Coop57 Madrid (2006), Coop57 Andalucía (2008) y Coop57 Galiza (2009). En todos estos casos, la clave del éxito en la constitución de una sección territorial de Coop57 ha sido la existencia de una red de economía social y solidaria en el propio territorio capaz de gestionarla.

Actualmente (30 de junio de 2012), Coop57 está integrada por 437 entidades socias y 2.117 personas ahorradoras (socias y socios colaboradores) y gestiona un volumen de ahorro de poco más de 13.000.000 euros y un saldo vivo de préstamos de 8.050.000 euros. Datos recientes de los movimientos de Coop57 son visibles en las Tablas 1 y 2.

A continuación destaco las principales características de la actividad y el funcionamiento que hacen de Coop57, una experiencia singular en el marco de las finanzas éticas.

\section{Autogestión}

Coop 57 funciona de forma completamente autogestionada. Entre todos los socios y socias, de forma democrática y participativa, decidimos qué hacemos y cómo lo hacemos, desde los criterios éticos y sociales hasta cómo se remunera cada año el ahorro y qué tipos de interés deben aplicarse a los préstamos, pasando por el sistema de garantías, los productos financieros, los planes de trabajo y los presupuestos anuales, etc.

Coop57 no se regula por las directrices del banco central, ni utiliza índices de referencia como el euríbor, sino por los acuerdos que adoptan colectivamente sus socios, y no está sujeta a regulaciones externas (más allá del marco jurídico que establece la Ley de cooperativas) ni a movimientos incontrolables o especulativos en el mercado de capitales. De esta forma se pone en práctica la democracia económica y se pueden establecer colectivamente unas reglas del juego estables y conocidas y compartidas por nuestras socias y socios. 


\begin{tabular}{|l|r|r|r|}
\hline \multicolumn{1}{|c|}{ Conceptos } & \multicolumn{1}{c|}{$\mathbf{2 0 1 2}$} & \multicolumn{1}{c|}{$\mathbf{2 0 1 1}$} & \multicolumn{1}{c|}{ Dif. \% } \\
\hline Capital fundacional & $178.180,05$ & $178.180,05$ & 0,00 \\
\hline Aportaciones obligatorias de las entidades socias & $407.699,38$ & $345.200,02$ & 18,11 \\
\hline Fondo de garantía de préstamos & $32.611,50$ & & \\
\hline Aportaciones voluntarias de las entidades socias & $793.704,29$ & $894.910,43$ & $-11,31$ \\
\hline Aportaciones de los socios y socias colaboradores & $14.476 .408,24$ & $9.366 .083,69$ & 54,56 \\
\hline Aportaciones transitorias & 0,00 & $34.385,00$ & $-100,00$ \\
\hline Total aportaciones socios & $\mathbf{1 5 . 8 8 8 . 6 0 3 , 4 6}$ & $\mathbf{1 0 . 8 1 8 . 7 5 9 , 1 9}$ & $\mathbf{4 6 , 8 6}$ \\
\hline Saldo vivo de préstamos concedidos a las entidades socias & $\mathbf{9 . 4 3 1 . 5 9 8 , 5 5}$ & $\mathbf{8 . 2 4 0 . 0 8 1 , 0 5}$ & $\mathbf{1 4 , 4 6}$ \\
\hline
\end{tabular}

Tabla 1. Datos económicos de Coop57 (2012).

Fuente: Elaboración propia.

Se trata de apropiarse colectivamente de dichas reglas del juego para no depender de los tristemente famosos mercados de capitales.

\section{Proximidad y confianza}

Toda la actividad que desarrolla Coop57 se basa en criterios de confianza: confianza entre

\begin{tabular}{|l|r|r|r|}
\hline & $\begin{array}{c}\text { Estmación ejercicio } \\
\mathbf{2 0 1 2}\end{array}$ & $\begin{array}{c}\text { Real ejercicio } \\
\mathbf{2 0 1 1}\end{array}$ & Dif. \% \\
\hline Ingresos & $\mathbf{5 9 7 . 0 2 5 , 0 0}$ & $\mathbf{4 8 5 . 0 0 3 , 3 8}$ & $\mathbf{2 3 , 1}$ \\
\hline Ingresos préstamos & $520.000,00$ & $423.253,50$ & 22,9 \\
\hline Intereses depósitos, c/c & $52.000,00$ & $21.552,94$ & 141,3 \\
\hline Otros ingresos de explotación & $25.025,00$ & $24.664,73$ & 1,5 \\
\hline Ingresos extraordinarios & 0,00 & $-690,79$ & $-100,0$ \\
\hline Ingresos encuentro & 0,00 & $16.223,00$ & $-100,0$ \\
\hline Gastos & $\mathbf{5 6 6 . 7 8 9 , 6 6}$ & $\mathbf{4 5 9 . 0 3 3 , 2 4}$ & $\mathbf{2 3 , 5}$ \\
\hline Personal & $166.480,10$ & $136.528,97$ & 21,9 \\
\hline Servicios Externos & $84.835,65$ & $59.461,33$ & 42,7 \\
\hline Dotaciones para amortizaciones & $16.000,00$ & $12.834,59$ & 24,7 \\
\hline Provisiones & $55.000,00$ & $46.452,16$ & 18,4 \\
\hline Remuneración aportaciones socios & $241.000,00$ & $180.779,28$ & 33,3 \\
\hline Gastos encuentro & 0,00 & $22.976,91$ & $-100,0$ \\
\hline Gastos extraordinarios & $3.473,91$ & 0,00 & 100,0 \\
\hline Resultados antes de impuestos & $\mathbf{3 0 . 2 3 5 , 3 4}$ & $\mathbf{2 5 . 9 7 0 , 1 4}$ & $\mathbf{1 6 , 4}$ \\
\hline
\end{tabular}

Tabla 2. Provisión de cierre del ejercicio 2012 (comparativa con el ejercicio 2011).

Fuente: Elaboración propia. 
los socios y socias, confianza de todas las personas que depositan sus ahorros en que serán gestionados adecuadamente, confianza en que las entidades que reciben préstamos harán un buen uso del dinero y lo devolverán de la forma pactada, etc. Sin esa base de confianza, una experiencia de finanzas éticas y solidarias nobancaria como Coop57 no sería posible. Si un proyecto económico con alto contenido social merece nuestra confianza, hacemos todo lo posible para concederle crédito, a pesar de que no cuente con garantías patrimoniales que respalden el préstamo. Confiamos en los proyectos y en las personas, no en su patrimonio.

Los criterios de confianza mutua se aplican al sistema de garantías de los préstamos. No utilizamos los mecanismos de garantías patrimoniales y solidarias al uso entre las entidades financieras convencionales, sino que aplicamos un sistema de garantías personales mancomunadas. Por ejemplo, si una cooperativa de intervención social pide un préstamo para hacer una inversión, pedimos a los miembros de la cooperativa y a su base social que avalen el préstamo mediante la firma de un documento en el que cada persona se compromete a retornar a Coop57 una determinada cantidad (nunca la totalidad del préstamo) en el caso de que la cooperativa no pueda hacerlo. Este documento es un compromiso asumido por cada persona, y no implica desembolsar dinero ni aportar o pignorar propiedades como garantía. Siempre buscamos que la entidad que pide el préstamo aporte el máximo de avales (incluso de personas no vinculadas directamente a la entidad) por dos motivos:

- Porque si la entidad es capaz de reunir muchos avalistas, es un indicativo de que genera confianza en su base social y su entorno y de que cuenta con un número de personas implicadas en el proyecto que responderá en el caso de que pase por apuros, reforzando así su viabilidad. Precisamente esa confianza es la que refuerza la confianza de Coop57. En el caso de que no genere confianza en su entorno inmediato y no sea capaz de reunir suficientes avales, generará dudas en Coop 57 respecto a la concesión del préstamo.

- Para que la cantidad avalada por cada persona sea lo más baja posible, de manera que sea razonable que pueda devolverla a Coop57 en el peor de los casos sin que hipoteque su vida y su economía personal.

Para todo ello, es fundamental la proximidad. No se pueden establecer relaciones de confianza desde la lejanía geográfica o la distancia social: hay que conocer bien las entidades, las personas que las integran, su entorno social y cultural, el impacto social de su actividad, etc. Eso sólo se puede hacer en situación de proximidad, y esta proximidad la garantiza nuestro funcionamiento en red.

\section{Flexibilidad}

La autogestión, las relaciones confianza y la proximidad a las entidades de economía solidaria comportan que seamos muy flexibles y podamos adaptarnos a sus necesidades. De esta forma, podemos ser muy imaginativos y ágiles a la hora de buscar soluciones a sus necesidades financieras. Si entre los socios nos ponemos de acuerdo para definir nuevos productos financieros, no tenemos que pedir permiso a nadie para ponerlos en práctica, pedir a notarios que den fe de nuestros acuerdos (más allá de lo que nos impone la legislación cooperativa), etc.

En Coop57 nunca hemos elaborado un producto y lo hemos lanzado posteriormente al mercado. Siempre los hemos definido a partir de las necesidades que nos han trasladado nuestros socios, tratando de no mimetizar lo que estaban haciendo los bancos convencionales.

\section{Funcionamiento en red}

Como he expuesto, el funcionamiento y el crecimiento descentralizado en red es una de las señas de identidad de Coop57. Las distintas secciones territoriales compartimos un mismo modelo de finanzas éticas, la misma forma jurídica y unos servicios centrales compartidos, aplicamos los mismos criterios de valoración éticos, sociales y económicos y ofertamos los mismos productos financieros, pero cada territorio autogestiona su base social y decide qué entidades admite como socias y qué préstamos se conceden. Además, el ahorro recogido en cada territorio se destina prioritariamente en conceder préstamos a entidades del propio territorio.

Además de posibilitar la proximidad y confianza explicadas en líneas precedentes, el fun- 
cionamiento en red también pretende evitar que, al crecer, Coop57 pierda su carácter participativo y autogestionado. Muchas veces los crecimientos comportan que las entidades y las cooperativas pierdan participación e implicación de sus socios, de manera que la democracia interna se resiente y los equipos técnicos y directivos cobran mayor autonomía respecto a la base social. Para evitarlo, se ha previsto que el mismo funcionamiento en red que permite crear secciones en nuevos territorios donde Coop57 no estaba implantada, sirva para «crecer hacia abajo», es decir, para descentralizar las secciones ya existentes. Actualmente ello se está materializando en la creación de grupos locales en algunas de las secciones territoriales, pero en un futuro puede comportar que una sección se desmiembre para dar lugar a nuevas secciones, tanto de ámbito territorial (por ejemplo, que abarque el Pirineo catalán) o de ámbito sectorial (que agrupe a las entidades de un mismo sector, por ejemplo las que se dedican a tareas de inserción socio-laboral de colectivos en riesgo de exclusión).

Otra característica fundamental es que la iniciativa de crear una nueva sección (sea territorial o sectorial, sea en un nuevo territorio o por desmembración de una sección ya existente) debe partir necesariamente de la voluntad del tejido asociativo y de economía solidaria del territorio o sector en cuestión. Debe partir de la autoorganización de personas y entidades que vean su utilidad, se apropien de ella y se comprometan a gestionarla, nunca de iniciativas impulsadas desde fuera.

\section{Líneas de futuro}

En el actual contexto de recesión económica y de restricciones financieras, la apuesta de Coop57 es mantener los servicios financieros para dar respuesta a las necesidades financieras de nuestros socios. A pesar de las dificultades económicas que atenazan a las entidades de economía social y solidaria, Coop57 debe seguir aumentando la concesión de préstamos para evitar que la falta de financiación acentúe su fragilidad.

También estamos haciendo una apuesta decidida por promover la financiación de nuevos proyectos cooperativos y de economía social que creen puesto de trabajo. En un contexto de paro desbocado y de acentuación de la crisis, creemos que debemos ser un agente activo en la promoción de la aparición de nuevos proyectos de economía solidaria. El mensaje es claro: dado que ni el sector público ni el privado crearán ocupación a corto y medio plazo, y puesto que hay que romper, en la medida que podamos, con el modelo económico que nos ha llevado a la actual crisis, tenemos que incentivar, mediante nuestra actividad financiera, que aparezcan y se consoliden nuevas experiencias que creen puestos de trabajo dentro de la economía solidaria aplicando modelos cooperativos.

Para poder afrontar los puntos anteriores de forma sostenible, además de aumentar los fondos propios de Coop57 y de reforzar las garantías de los préstamos, hemos optado por desarrollar sinergias con otras entidades para compartir riesgos y, así, poder multiplicar los servicios de financiación para las entidades de economía social y solidaria. En Coop57 contamos con recursos para poder conceder préstamos, pero queremos compartir los riesgos que conllevan con todas aquellas personas y entidades interesadas en desarrollar la economía solidaria. Es una línea que está dando resultados, desde la colaboración con la sociedad de garantía recíproca Oinarri, hasta la firma de convenios de colaboración con entes locales para promover nuevos proyectos cooperativos, pasando por la organización del I Concurso de Empresariado Social y Cooperativo en Santa Coloma de Gramenet (Barcelona) o la creación del Fondo Social Germinem en Reus (Tarragona) para la promoción de la ocupación, la economía solidaria y el asociacionismo popular en dicha localidad.

Siguiendo la apuesta por establecer sinergias con otras entidades, Coop57 también trabaja para contribuir a la articulación de un sistema de finanzas éticas que dé respuestas diversificadas al conjunto de necesidades de los ahorradores responsables y de las entidades de economía social y solidaria, que incluya desde iniciativas no-bancarias, como Coop57, a proyectos bancarios, pasando por entidades dedicadas a hacer préstamos solidarios o por grupos de ayuda mutua en el ámbito financiero o el sector de los seguros éticos y solidarios. En este sentido, participa en el proyecto FIARE para promover el establecimiento en España de un banco ético participativo, siguiendo el modelo 
de la Banca Popolare Etica, es miembro de varias redes y entidades de promoción y coordinación de los instrumentos de finanzas éticas y cooperativas, como FETS o FINANCOOP, y tiene establecidos convenios de colaboración con FIARE y la correduría de seguros ARÇ.

Otra línea de trabajo en marcha es profundizar la capilaridad social y la descentralización de Coop57. En esta línea, están surgiendo en varias secciones y grupos locales con la finalidad de dinamizar la base social, desarrollar tareas de difusión, sensibilización y extensión y promover en el ámbito local la economía solidaria. En todos los casos, la creación de grupos locales surge por iniciativa de los propios socios y socias de Coop57 del territorio. Estos grupos locales también son fundamentales para profundizar la proximidad y confianza que caracteriza la relación de Coop57 con sus socios.

Una última línea de futuro que estamos empezando a poner en marcha es la promoción del mercado social, es decir una red de intercambio de bienes y servicios entre entidades de la economía solidaria y las personas consumidoras responsables, de forma que en ella puedan cubrir una parte significativa de sus necesidades, sin tener que recurrir al mercado capitalista. En la medida que Coop57 agrupa a un número significativo de personas y de entidades vinculadas con la economía solidaria, tenemos que aprovechar este potencial para, más allá de nuestra actividad financiera, promover dicho mercado social. Es una línea incipiente, en la que estamos colaborando con las iniciativas promovidas por las distintas redes de economía solidaria del Estado.

\section{El Trabajo Social y las finanzas éticas y solidarias}

En la mesa redonda «Finanzas éticas para la transformación del mundo», celebrada el pasado 14 de marzo en la Universidad Pontificia de Comillas en el marco del IX Foro de Trabajo Social, su coordinador, Carlos Ballesteros, nos planteó a los ponentes qué les pediríamos a los trabajadores sociales. A pesar de que no soy un especialista en la materia, me atrevo a apuntar algunos elementos.

Como he explicado, en Coop57 estamos convencidos de que el objetivo de las finanzas éticas debe ser transformar la sociedad, construyendo un modelo económico basado en los principios y la práctica de la economía solidaria. Las finanzas éticas deben ser uno de los medios que posibiliten la consecución de este objetivo. Según la Federación Internacional de Trabajadores y la Asociación Internacional de Escuelas de Trabajo Social (IASSW), el Trabajo Social es la disciplina que promueve el cambio social, la resolución de problemas en las relaciones humanas, y el fortalecimiento y la liberación del pueblo, para incrementar el bienestar (Wikipedia). Por lo tanto, las finanzas éticas y el Trabajo Social pueden (y deben) compartir el objetivo de transformar la sociedad y de incrementar el bienestar de las personas.

Uno de los retos actuales para paliar los problemas sociales es la revitalización del tejido económico y la creación de puestos de trabajo que tengan calidad y estabilidad, pero mediante modelos económicos que pongan en primer lugar la satisfacción de las necesidades de las personas, la equidad, la participación, la cohesión social y el respeto al medio ambiente, es decir, que nos permitan transformar la sociedad.

Las trabajadoras y los trabajadores sociales pueden contribuir a ello de muchas formas distintas: impulsando la creación de cooperativas de intervención social; promoviendo que los colectivos desfavorecidos o en riesgo de exclusión social monten sus propios puestos de trabajo autogestionados; colaborando desde su práctica profesional con las entidades que se dedican a fomentar la economía solidaria (incluidas las entidades de finanzas éticas); etc. En Coop57 constatamos que hay muchos profesionales de Trabajo Social que están comprometidos con entidades de economía solidaria que promueven el desarrollo comunitario, la inserción socio-laboral, las actividades de ocio colectivo no-consumistas, etc.

Básicamente se trata de tener presente que la economía solidaria es una alternativa válida e imprescindible para transformar la sociedad. Y, para ello, el primer paso es conocerla; en consecuencia, es fundamental que desde la actividad docente se difundan sus principios y su práctica (y, también, los de las finanzas éticas) y que las entidades representativas de las trabajadoras y los trabajadores sociales las den a conocer. En esta dirección, gestos como la incorporación como socio colaborador a Coop57 del Colegio Oficial de Trabajo Social de Cata- 
lunya, efectuando una aportación de ahorro, son importantes.

La crisis actual nos abre la oportunidad de promover, aunque sea a pequeña escala, un cambio de modelo económico y social: todas las grandes transformaciones sociales empiezan con iniciativas pequeñas y minoritarias que desbrozan el camino a seguir. En nuestras ma- nos está hacerlo posible. Una solución equitativa a la crisis y a sus consecuencias sociales no caerá del cielo, únicamente podemos promoverla colectiva y cooperativamente, generando una economía alternativa. Las múltiples iniciativas de economía solidaria y de finanzas éticas demuestran que no sólo es necesario, sino que es posible.

\section{Referencias bibliográficas}

Alsina, O. (coord.) (2004). La banca ética: Mucho más que dinero. Barcelona: Icaria.

Artime, D. (2012). Banca ética: Dinero contra el sistema. Atlántica XXII: Revista Asturiana De Información y Pensamiento, 18, 43-44.

Ballesteros García, C. (2004). Experiencias de banca ética en Europa. En El día de Europa. Las transformaciones de la Unión Europea: La ampliación y la convención europea (pp.143-158). Madrid: Universidad Pontificia de Comillas.

Bicciato, F. (2002). Financiación del tercer sector en Italia: Banca popolare etica. En La banca ética : Mucho más que dinero (pp. 25-33). Barcelona: Icaria.

Boleda, I. (2004). Banca ética: mucho más que el dinero. Barcelona: Oikocredit.

Comune di Roma. (2004). Cosa è l'altra economía. Roma: Comune di Roma.

Coriat, B. (1997). Los desafios de la competitividad. Buenos Aires: Universidad de Buenos Aires

Cortés García, F. (2012). La inversión socialmente responsable y la banca ética. Alternativas: Revista de Análisis y Reflexión Teológica, 43, 225-234.

Cruz Ayuso, C. de la, y Sasia Santos, P. M. (2010). La banca ética como herramienta de acción política. Icade:Revista de las Facultades de Derecho y Ciencias Económicas y Empresariales, 80, 185-210.

Cruz, C. de la, Sasia Santos, P. M. y Garibi, J. (2006). Lo que debe ser y es una banca ética. Lan Harremanak: Revista de Relaciones Laborales, 14, 175-204.

Cruz, C. de la y Sasia, P. M. (2008). Banca ética y ciudadanía. Madrid: Editorial Trotta, S.A.

Cuesta González, M. de la (2002). Algunas alternativas en el sistema financiero: La banca ética. En J. I. Flor (coord.), Globalización, crisis ambiental y educación (pp. 215-238). Madrid: Ministerio de Educación, Subdirección General de Información y Publicaciones.

Cuesta González, M. de la, Fernández Olit, B. y Vázquez Oteo, O. (2006). Ensayos: razón de ser de la banca ética. Experiencias internacionales y nacionales. Madrid: Fundación de las Cajas de Ahorros

Del Río Paracolls, N. (2003). Rescata tu dinero, finanzas solidarias y transformación social. Madrid: Talasa Ediciones.

Gasch, A. (2009). Fiare banca ètica: Estalvis solidaris per canviar el món. Poblet, 2(17), 80.

Gómez Sanz, M. (2005). Dinero con compromiso: Triodos bank introduce en España el nuevo modelo de banca ética. Emprendedores: Las Claves de la Economía y el Éxito Profesional, 91, 34-38.

Guerra, P. (2002). Socioeconomía de la Solidaridad. Montevideo: Nordan.

Guerra, P. (2012). Miradas globales para otra economía. Cataluña: Setem.

Ibáñez Jiménez, J. W. (2004). Responsabilidad social de la empresa y finanzas sociales. Madrid: Ediciones Akal.

Kennedy, Margritt (1998). Dinero sin inflación ni tasas de interés. Buenos Aires: Nuevo Extremo. Llobet, M. (2002). La banca ètica. Papers de Joventut: Informatiu, 39, 13-13.

Mateos, N. (2011). Canviar el món amb els nostres estalvis: La banca ètica. Foc Nou, 445, 36-37. Melé, J. A. (2010). Banca ética para transformar el mundo. Crítica, 60(970), 73-76.

Milano, R. (2001). La finanza e la banca ética. Economia e solidarità. Roma: Edizioni Paoline.

Panyella Carbonell, J. (2008). ¿Qué hacer con mi dinero?: Finanzas éticas: Una propuesta para usar el dinero en función de lo que quiero. Cañamo: La Revista de la Cultura del Cannabis, $122,32-34$. 
Passini, M. (2005). Banca ética: Experiencias internacionales. el caso italiano. En M. de la Cuesta González y A. Galindo García, Inversiones socialmente responsables (pp. 283-304). Salamanca:Universidad Pontificia de Salamanca.

Puyo, A. (2007). Banca ética, una alternativa de futuro. Éxodo,91, 47-48.

Puyo, A. (2007). FIARE, un proyecto de banca ética. En La Calle: Revista sobre situaciones de Riesgo Social, 8, 26-28.

Río, N. d. (2011). Banca ética y transformación social. Papeles de Relaciones Ecosociales y Cambio Global, 114, 69-79.

San José Ruiz de Aguirre, L. y Retolaza Avalos, J. L. (febrero, 2007). Análisis comparativo de la banca ética con la banca tradicional: Identificación de indicadores. En Grupo de Investigación FEDRA, XVII Jornadas Hispano Lusas. Conocimiento, innovación y emprendedores: Camino al futuro. Logroño: Universidad de La Rioja.

Sánchez-Matamoros Fontenla, M., y Jiménez Soto, M. A. (2009). Banca ética y transformación social. En Economía y desarrollo humano: Visiones desde distintas disciplinas (pp. 179-206). Granada: Universidad de Granada.

Sasia Santos, P. M. (2008). Entender hoy la banca ética. Revista Internacional de los Estudios Vas$\cos , 53(2), 507-532$. Recuperado en = Eusko Ikaskuntzen Nazioarteko Aldizkaria = Revue Internationale Des Études Basques = International Journal on Basque Studies,

Sasia Santos, P. M. (2012). La banca ética en Europa: El enfoque del crédito como criterio de configuración de un espacio de alternativa. CIRIEC - España .Revista de Economía Pública, Social y Cooperativa, 75, 277-300.

Sasia Santos, P. M., y Garibi, J. (2012). Análisis de los perfiles y desafíos de la banca ética desde la experiencia del proyecto fiare. Boletín de Estudios Económicos, 67(206), 289.

Sasia, P. (2007). Banca ética: Intermediación financiera al servicio de la justicia. Crítica, 57(942), 76-79.

Sasia, P. M. (2013). ¿Es la banca ética una alternativa a la crisis? Crítica, 983, 35-39.

Serrano, A. (2009). Banca ética: La otra cara de la moneda. Monitor Educador, 131, 44-45.

Setem (Septiembre 2009). Guía sobre finanzas éticas para ahorradores: Tú y el dinero. Barcelona: Setem

Sunyer, R., y Alsina, O. (2001). Quan hi haurà una banca ètica a catalunya? Àmbits De Política $i$ Societat, 16, 7-9.

Sunyer, R. y Alsina, O. (1999). La banca ètica: Una experiència consolidada. Diàlegs: Revista d'Estudis Polítics $i$ Socials, 2(4), 143-162.

Teis, X. (2011). Cómo cambiar el mundo con tu dinero, alternativas a la banca convencional. Barcelona: Icaria.

Teis, X. (2012). Finanzas éticas: Economía y personas. Economía Exterior: Estudios de la Revista Política Exterior sobre la internacionalización de la Economía Española, 60, 108-114.

Toña Guenaga, A., Sasia, P. y Garibi, J. (2006). Posibilidades de estructuración de una banca ética en España. Documentación Social, 142, 157-174.

Torreguitart Mirada, M. C. y Estapé Dubreuil, G. (2011). El proyecto de finanzas éticas y solidarias seguido por la cooperativa Coop57. Revista Española del Tercer Sector, 19, 121-142.

Tostado, P. (2011). Propuesta del proyecto de banca ética fiare. Éxodo, 107, 53-54.

Vidal, P. , De la Peña Rodríguez, M., Zaragoza, M. y Sureda, M. (2006). Diagnóstico sobre el interés del tercer sector y la economía social por una banca ética. Barcelona: FETS. 